\title{
Experimental Methods to Quantify Bentonite Swelling Anisotropy
}

\author{
ALEX HoINVILLE, KATHERINE TELFEYAN*, HAKIM \\ BOUKHALFA
}

Los Alamos National Laboratory, Los Alamos, NM 87544, USA (*Correspondence: ktelfeyan@lanl.gov)

Bentonite clays are considered as additional containment barriers in the designs of deep geologic disposal concepts for high level radioactive waste (HLRW). Several properties make bentonite a candidate barrier material for HLRW disposal in geologic repositories. When compressed bentonite has a very low permeability, it has a high cation exchange capacity, reduces biofilm development, and it swells significantly on exposure to water. The swelling properties of bentonite are of interest due to the ability of bentonite to swell into and seal gaps between the waste container, barrier, and host rock. Recent efforts in analytical modelling of the swelling expansion of bentonite clay indicate that the swelling of bentonite at a quick rate, such as swelling bentonite in low ionic strength water, can introduce anisotropy in the swelling. Anisotropy of swelling in low ionic strength water may have an effect on barrier erosion and potential for radionuclide transport from the repository and should be considered in safety assessment.

In order to investigate the swelling properties of Wyoming MX80 bentonite, a millimetre-scale batch swelling method was devised, introducing water to small compressed bentonite pellets in semi-constrained and unconstrained conditions, taking photographs of the clay through a microscope at regular intervals as it swells, and using visual image analysis techniques to analyse the resulting images. Swelling tests in solutions with increasing $\mathrm{NaCl}$ and $\mathrm{CaCl}_{2}$ concentrations between $0.02 \mathrm{mMolL}^{-1}$ and $200 \mathrm{mMolL}^{-1}$ were performed. Image analysis was performed in the open-source program ImageJ and involved threshold analysis for area, and processing to extract shape indices. In the semi-constrained experiments, the bentonite pellets were constrained vertically so that they could only swell in two dimensions, removing vertical swelling as a factor. This batch experiment-image analysis method is well suited to investigating potential anisotropy in swelling due to the small clay volume, short swelling timescales, ease of visual evaluation of isotropyanisotropy, and the ease of which the image data feeds back into models of bentonite swelling. Changes in size over time were recorded alongside a shape descriptor in order to compare the shape of the pellet with the swelling rate. Data is presented illustrating the observed relationship between swelling rate and shape of the bentonite pellet, used as a proxy for the anisotropy of the swelling. 